\title{
A narrative review on the interaction between genes and the treatment of hypertension and breast cancer
}

\author{
Wenjuan Wang ${ }^{1 \#}$, Qingjian He ${ }^{2 \#}$, Haodong Zhang ${ }^{1}$, Chenchen Zhuang ${ }^{1}$, Qiongying Wang ${ }^{1}$, Caie Li $^{1}$, \\ Runmin $\operatorname{Sun}^{1}$, Xin Fan ${ }^{1}$, Jing $\mathrm{Yu}^{1}$ \\ ${ }^{1}$ Department of Hypertension Center, Lanzhou University Second Hospital, Lanzhou, China; ${ }^{2}$ Department of Breast and Thyroid Surgery, \\ Zhoushan Hospital of Zhejiang Province, Zhoushan, China \\ Contributions: (I) Conception and design: W Wang; (II) Administrative support: J Yu; (III) Provision of study materials or patients: J Yu; (IV) \\ Collection and assembly of data: Q He; (V) Data analysis and interpretation: W Wang; (VI) Manuscript writing: All authors; (VII) Final approval of \\ manuscript: All authors. \\ \#These authors contributed equally to this work. \\ Correspondence to: Jing Yu. Department of Hypertension Center, Lanzhou University Second Hospital, No. 82 Cuiyingmen, Chengguan District, \\ Lanzhou, China. Email: ery_jyu@lzu.edu.cn.
}

\begin{abstract}
Objective: The aim to discuss the close relationship between the common biological mechanisms of breast cancer and hypertension, inflammation and oxidative stress, breast cancer gene mutations breast cancer susceptibility gene (BRCA), G protein-coupled receptor kinase (GRK4), etc. and breast cancer treatment includes chemotherapy, Endocrine therapy, Targeted therapy and anti-angiogenesis drugs. In antiangiogenesis drugs focusing on the mechanism of tyrosine kinase inhibitors (TKI) that may activate the rhoa/ rock pathway to cause hypertension, as well as the relationship between breast cancer and antihypertensive drugs includes angiotensin-converting enzyme inhibitors (ACEIs), Calcium channel blockers (CCBs) and $\beta$-blockers (BBs)will be explored.

Background: Cardiovascular diseases (CVD) and tumors are the two major types of diseases with the highest mortality rates, while hypertension accounts for the largest proportion of CVDs. A large number of the same or similar risk factors are shared between hypertension and tumors, and they influence each other. Many patients, particularly elderly patients, often present with the coexistence of the two diseases. As medical advances have enabled clinicians to cure tumors, many patients with cancer live longer, leading to a gradual increase in the incidence of CVDs, including hypertension. With the second highest incidence among tumors, breast cancer has gradually attracted widespread attention and has been the topic of numerous studies. Studies have confirmed that CVD is one of the causes of death in elderly patients with breast cancer. Methods: Publications from 1985 to 2020 were retrieved from the Web Of Science, Cochrane Library, PubMed, EMBASE and MEDLINE database. We used a mix of MeSH and keywords.

Conclusions: Hypertension and cancer may share a common mechanism. The screening and risk assessment of breast cancer in patients with hypertension must be strengthened. Breast cancer cardiology is the interdisciplinary study of oncology and cardiology, and in-depth research in this field may result in long-term improvements in the survival and prognosis of patients with both clinical hypertension and breast cancer.
\end{abstract}

Keywords: Hypertension; breast cancer; tumor treatment; hypertension treatment

Submitted Mar 15, 2021. Accepted for publication May 13, 2021.

doi: 10.21037/atm-21-2133

View this article at: http://dx.doi.org/10.21037/atm-21-2133 


\section{Introduction}

Hypertension is the largest contributor to the burden of cardiovascular disease (CVD), and the prevalence of the disease is increasing in different regions (1). According to a survey, the prevalence of CVD in China is continuously increasing. The estimated number of patients with CVDs is 290 million, and the estimated number of patients with hypertension is 245 million. In other words, the incidence of hypertension accounts for $84.48 \%$ of CVD. Moreover, the control rate and treatment compliance rate of hypertension are still very low. With the continuous improvement of medical technology, the life span of patients with cancer has been extended, and millions of patients with various cancers have survived to date. However, the progress in treatment has increased the overlap between cancer and CVD, and these patients have a risk of developing CVD $(2,3)$.

Although CVD and cancer are generally considered two separate disease entities, they share various risk factors and possible interactions (Figure 1). Hypertension is a CVD with a very high prevalence rate, and it is also an important cardiovascular risk factor. A study presented at the European Multidisciplinary Cancer Conference in 2011 suggested that men whose blood pressure is higher than normal have a significant increase of $10-20 \%$ in the risk of cancer, and both men and women have an increased risk of dying from cancer. In recent years, many studies have also found an association between tumors and hypertension $(4,5)$. These diseases may be causal factors for each other, and increasing evidence confirms a close relationship between the two (Figure 1). Studies exploring the relationship between hypertension and tumors as well as the mechanism underlying their co-occurrence will have great benefits for clinical work.

We present the following article in accordance with the Narrative Review reporting checklist (available at http:// dx.doi.org/10.21037/atm-21-2133).

\section{Literature search methods}

Publications from 1 January 1985 to 31 December 2020 were retrieved from the Web Of Science, Cochrane Library, PubMed, EMBASE and MEDLINE databas, without any language restrictions. We used a mix of $\mathrm{MeSH}$ and keywords. With the following terms: Hypertension OR (Blood Pressure, High) OR (Blood Pressures, High) (High Blood Pressure) OR (High Blood Pressures) AND (Breast Neoplasm) OR (Neoplasm, Breast) OR (Breast Tumors) OR
(Breast Tumor) OR (Tumor, Breast) OR (Tumors, Breast) OR (Neoplasms, Breast) OR (Breast Cancer) OR (Cancer, Breast) OR (Mammary Cancer) OR (Cancer, Mammary) OR (Cancers, Mammary) OR (Mammary Cancers) OR (Malignant Neoplasm of Breast) OR (Breast Malignant Neoplasm) OR (Breast Malignant Neoplasms) OR (Malignant Tumor of Breast) OR (Breast Malignant Tumor) OR (Breast Malignant Tumors) OR (Cancer of Breast) OR (Cancer of the Breast) OR (Mammary Carcinoma, Human) OR (Carcinoma, Human Mammary) OR (Carcinomas, Human Mammary) OR (Human Mammary Carcinomas) OR (Mammary Carcinomas, Human) OR (Human Mammary Carcinoma) OR (Mammary Neoplasms, Human) OR (Human Mammary Neoplasm) OR (Human Mammary Neoplasms) OR (Neoplasm, Human Mammary) OR (Neoplasms, Human Mammary) OR (Mammary Neoplasm, Human) OR (Breast Carcinoma) OR (Breast Carcinomas) OR (Carcinoma, Breast) OR (Carcinomas, Breast). The final reference list was generated on the basis of relevance and originality with regard to the topics covered in this Review.

\section{The relationship between breast cancer and hypertension (Figure 1)}

Hypertension is a common chronic disease and a major risk factor for cardiovascular and cerebrovascular diseases and chronic kidney disease. Breast cancer is the second most common cancer in the world (1.7 million cases) and the fifth leading cause of death worldwide in 2012 (522,000 cases) (6). Arterial hypertension is closely associated with renal cell cancer development (7). Hypertension is also one of the risk factors for breast cancer, and the prevalence of hypertension and breast cancer in women increases with age (8). In particular, the risk of breast cancer is more significantly increased in postmenopausal women than in premenopausal women $(9,10)$. Similarly, postmenopausal women with hypertension have twice the risk of breast cancer as nonhypertensive women, and postmenopausal estrogen withdrawal may be one of the causes of postmenopausal hypertension (11). One of the reasons may be the changes in estrogen levels. After menopause, the body's endocrine is prone to changes. In addition, the lower basal metabolic rate makes the body fat. The combined influence of these factors will indeed increase the prevalence of breast cancer. Other possible mechanisms require further research and exploration. The effect of postmenopausal breast cancer on CVD in elderly women is not yet clear. Compared with 


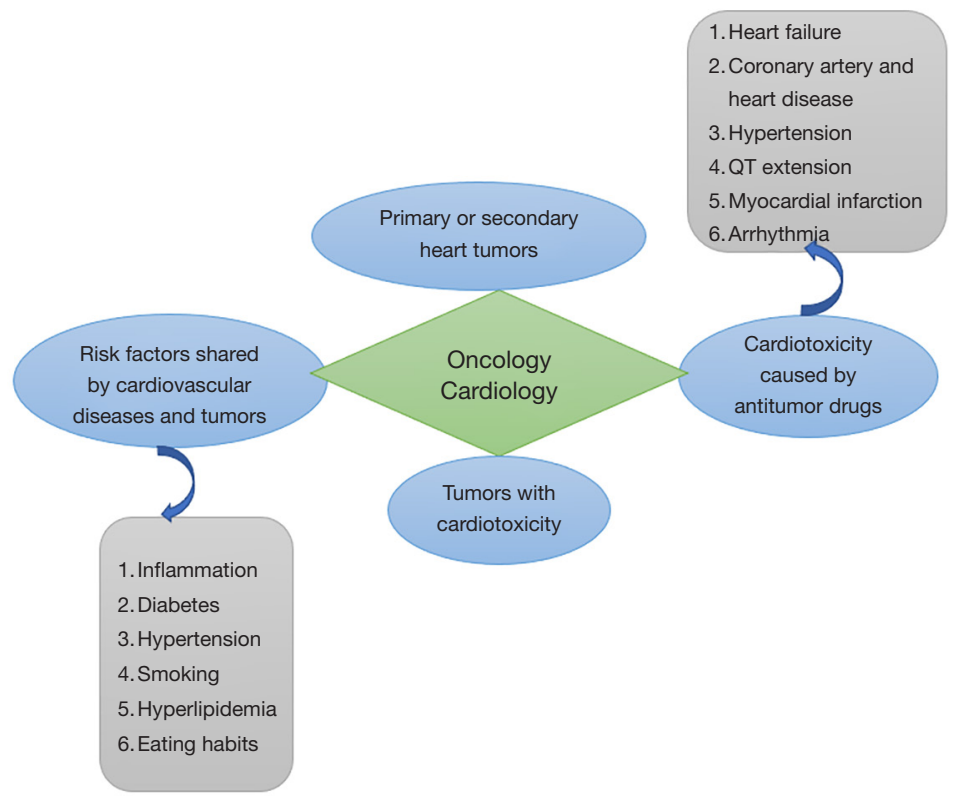

Figure 1 The subject orientation of oncology and cardiology.

elderly women without breast cancer, postmenopausal elderly women with breast cancer have a higher risk of CVD after an average of 10 years of follow-up. Only 17\% of women die of breast cancer, whereas CVD is the leading cause of death (22\%) (12). Therefore, among patients with breast cancer, CVD is more likely to cause death in breast cancer patients than in patients without CVD. That is, breast cancer patients with CVD risk factors have a higher mortality rate than patients without CVD.

From 1970 to 2014, the growth rate of the incidence of breast cancer among Chinese women was more than four times the growth rate in the US population (13), seriously affecting national health and social resources. A family history of breast cancer, obesity, and lack of exercise are considered risk factors for breast cancer $(14,15)$. Similarly, obesity and a lack of exercise are also risk factors for hypertension. After controlling for obesity factors, hypertension and breast cancer are still strongly correlated. The risk of breast cancer is consistently associated with a higher BMI, and BMI is related to high blood pressure (16). In addition, the steroid hormone estradiol, which is a preventive factor for patients with hypertension, is also involved in the development of breast cancer (8). Metaanalyses showed a positive correlation between hypertension and breast cancer, but the specific mechanisms are not yet clear $(9,17)$.

Our previous meta-analysis found a close relationship between hypertension, antihypertensive drugs, and breast cancer (18). The incidence of hypertension in patients with breast cancer increases with age. Estrogen can delay the increase in blood pressure and participate in the development of breast cancer. The occurrence and transition of the disease is related to inflammatory mediators and angiogenesis (18).

The 2018 American Heart Association (AHA) Scientific Statement: Cardiovascular Disease and Breast Cancer stated that CVD and breast cancer have many common risk factors, such as age, diabetes, family history, drinking, hormone replacement therapy, obesity/overweight, physical activity, and smoking, amongst others. Related research shows that breast cancer treatment, particularly mediastinal and left breast cancer radiotherapy, anthracycline chemotherapy, trastuzumab, and antiangiogenic drugs, may increase the cardiovascular risk of patients with breast cancer.

\section{The common biological mechanisms of breast cancer and bypertension involve inflammation and oxidative stress}

The levels of inflammatory factors, including TNF- $\alpha$, IL-6, and high sensitivity $\mathrm{C}$ reactive protein (hsCRP), are closely related to the occurrence and development of hypertension. Inflammatory cytokines play an important role in the development of disease in patients 


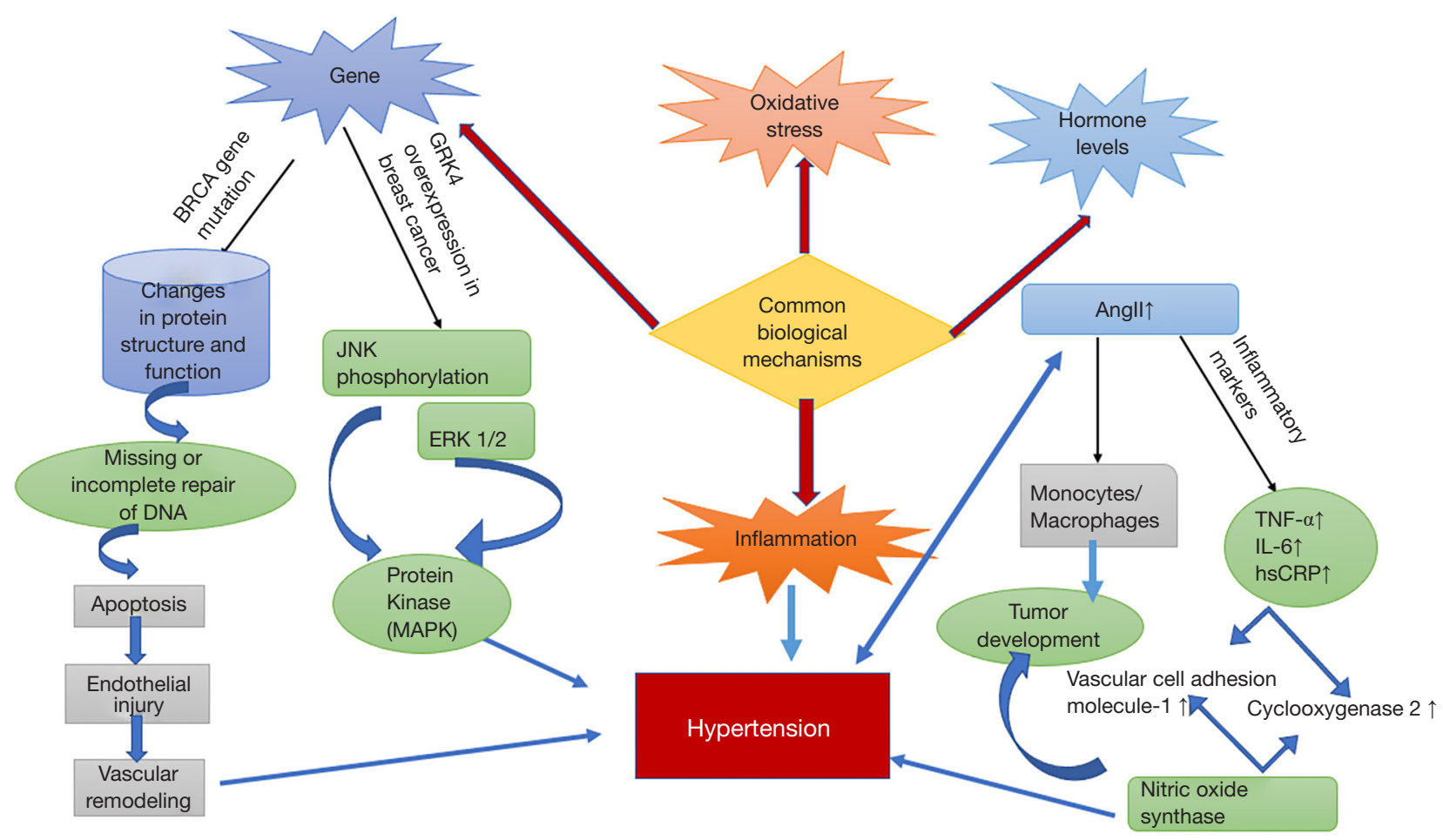

Figure 2 The common biological mechanisms of breast cancer and hypertension include chronic inflammation stimulation, oxidative stress, changes in hormone levels, and the relationship between breast cancer, hypertension and gene mutations including BRCA, and GRK4. BRCA, breast cancer susceptibility gene; GRK4, G protein-coupled receptor kinase; JNK, c-Jun N-terminal kinase; ERK1/2, extracellular regulated protein kinases 1/2; MAPK, mitogen-activated protein kinase; AngII, Angiotensin II; TNF- $\alpha$, tumor necrosis factor- $\alpha$; IL-6, Interleukin-6; hsCRP, high sensitivity $\mathrm{C}$ reactive protein.

with hypertension, and the condition of patients with hypertension directly impacts the levels of inflammatory factors. Angiotensin II-induced hypertension increases the levels of markers of aortic monocytes/macrophages and aortic inflammation by increasing the mRNA expression of vascular cell adhesion molecule-1, cyclooxygenase 2, and inducible molecules (19) (Figure 2). The study by Zahid et al. showed that the resultant state of chronic lowgrade inflammation is associated with the activation of NF$\kappa \mathrm{B}$ signaling and elevated levels of aromatase, the ratelimiting enzyme in estrogen biosynthesis. This occurs not only in the visceral and subcutaneous fat, but also in the breast fat. The regulation of aromatase in the breast adipose stromal cell in response to inflammatory mediators is under the control of complex signaling pathways, including metabolic pathways involving LKB1/AMPK, p53, HIF1 $\alpha$ and PKM2 (20). The role of inflammation in promoting cancer has been confirmed (3). Inflammation in the tumor microenvironment promotes malignant transformation, carcinogenesis, and the progression of cancer (21). On the other hand, increasing evidence shows that oxidative stress and its immediate consequences, including lipid peroxidation, are associated with multiple pathological conditions, such as atherosclerosis, cancer, and inflammation (Figure 2). Biological systems are continuously exposed to oxidants, whether they are derived from endogenous metabolic reactions or exogenous sources (such as toxins and smoking), and oxidative stress is caused by the imbalance of oxidants and antioxidants. Chronic inflammation, CVD, and common causes of cancer (such as diabetes, high blood pressure, and obesity) also cause oxidative stress (22).

Hypertension causes inflammation, and inflammation potentially promotes the occurrence and progression of breast cancer. Inflammation in the breast cancer microenvironment promotes the malignant transformation of cells and carcinogenesis, and accelerates the progression of breast cancer, forming a vicious cycle. Other factors contributing to inflammation may also include hormones, 
particularly in menopausal women, who have a higher risk of breast cancer and high blood pressure, which may also be related to changes in hormone levels in the body. In addition, growth factors and cytokines are also related factors that cause hypertension and breast cancer, and a large number of experiments are currently needed to examine their roles in these diseases.

\section{Breast cancer gene mutation and hypertension}

\section{BRCA gene mutation}

The lifetime risk of breast cancer in women in Western countries is $12 \%$, the cumulative risk of breast cancer at the age of 70 years for BRCA1 mutation carriers is $57-65 \%$, and the corresponding cumulative risk of BRCA2 carriers is $45-49 \%(23,24)$. After excluding the death of patients with BRCA-related malignant tumors, the average life span of carriers with BRCA1/2 mutations is still reduced (25). Compared with the general population, people with mutations in the BRCA1/2 genes have a higher risk of CVD (26). The exact mechanism has not yet been elucidated, and further experiments are needed. BRCA mediates DNA damage repair and reduces the generation of reactive oxygen species (ROS). It is a protective factor for atherosclerosis. The BRCA gene plays a role in maintaining the integrity of the genome by participating in the cellular response to DNA damage. BRCA mutations lead to changes in the structure and function of the corresponding protein, causing a loss of DNA repair or incomplete repair, thereby inducing apoptosis, a process that occurs in all types of cells including vascular endothelial cells and Tumor Cell (27). This altered DNA repair of BRCA mutations may also be a cause of endothelial damage, vascular remodeling, and increased blood pressure in patients with hypertension (Figure 2).

\section{The role of G protein-coupled receptor kinase (GRK4) in breast cancer and bypertension}

GRKs include 7 mammalian serine/threonine protein kinases that are regulated by an oncogene called c-Myc, which is overexpressed in breast cancer and plays a role in many other cancers. The GRK4 protein is present in breast cancer cells, but not in normal breast cells. In addition, GRK4overexpressing cells phosphorylate extracellular regulated protein kinases $1 / 2$ (ERK 1/2) and c-Jun N-terminal kinase (JNK) to activate mitogen-activated protein kinase (MAPK) (28). Based on these results, GRK4 may be associated with breast cancer carcinogenesis (Figure 2).

In addition, compared to women with normal blood pressure, women with hypertension have an approximately $15 \%$ increase in the risk of breast cancer. High levels of GRK4 have been shown to cause high blood pressure (29). A meta-analysis showed an association of the rs 1801058 polymorphism in the GRK4 gene with hypertension in East Asian and European people. Among Europeans, the rs2960306 polymorphism in the GRK4 gene was also found to be significantly associated with hypertension (30). These studies further confirmed the relationship between the GRK4 gene and hypertension, but the specific mechanism remains unclear and further experiments are needed. Therefore, GRK4 may be a risk factor for hypertension and breast cancer. A drug targeting GRK4 may be used to treat patients with hypertension and breast cancer in the future.

\section{Related mechanisms of hypertension caused by breast cancer treatment}

The treatment of breast cancer is divided into local treatment and systemic treatment. Local treatment includes surgical treatment and radiotherapy, the purpose of which is to kill local tumor cells. Systemic treatment includes chemotherapy, endocrine therapy, and molecular targeted therapy. These treatments all involve the occurrence of cardiovascular events. In patients with breast cancer, cancer treatment may increase the risk of CVD through direct or indirect mechanisms. Chemotherapy, and radiotherapy directly induce structural damage to the cardiovascular system, while endocrine therapy may indirectly affect certain risk factors for CVD $(31,32)$ (Figure 3).

\section{Chemotherapy}

The anthracycline cardiolipin has a higher affinity, enters mitochondria, inhibits the respiratory chain, and causes myocardial damage. Moreover, the heart lacks catalase, meaning that the antioxidant activity is weakened, and myocardial cells are rich in mitochondria, the source of ROS (Figure 3). Therefore, the cardiotoxicity induced by anthracycline chemotherapy includes (I) oxygen free radical damage theory, $\mathrm{OH}-, \mathrm{O}_{2}$ and anthracycline-Fe3+ complex; (II) calcium overload and energy metabolism theory, activate sarcoplasmic reticulum $\mathrm{Ca} 2+$ channel and inhibit $\mathrm{Ca} 2+-$ ATP enzyme expression; (III) iron ion disorder theory, ferritin is reduced, active $\mathrm{Fe} 2+$ is released, participate in the reaction to produce free radicals. 


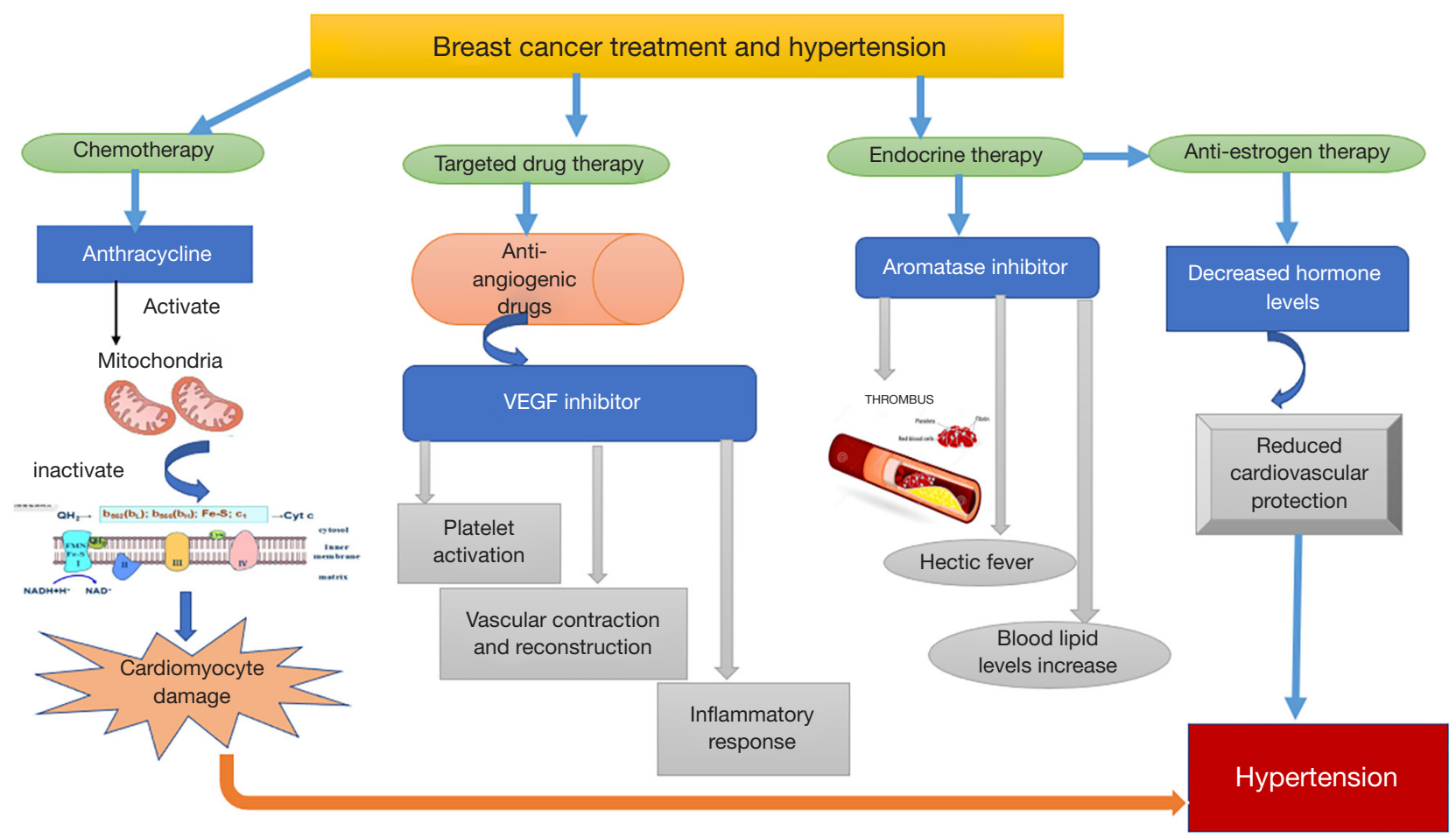

Figure 3 Related mechanisms of hypertension caused by breast cancer treatment, includes chemotherapy, endocrine therapy, and molecular targeted therapy. VEGF, Vascular endothelial growth factor.

\section{Endocrine therapy}

Patients with early breast cancer who are estrogen receptor (ER)-positive and/or progesterone receptor (PR)-positive have a better prognosis after receiving adjuvant endocrine therapy. Because more effective treatment extends the life span of these patients, they are more likely to die from CVD (including coronary heart disease and cerebrovascular disease), not breast cancer (33). Currently, the commonly used drugs for breast cancer endocrine therapy are antiestrogen drugs, aromatase inhibitors, progestins, and luteinizing hormone-releasing hormone analogs (LHRHa), amongst others (Figure 3).

\section{Aromatase inhibitors}

Aromatase inhibitors still cause adverse reactions, such as thromboembolism, hot flashes, and increased blood lipid levels, which can further increase the risk of hypertension.

\section{Antiestrogen drugs}

Tamoxifen (TAM) is currently the most commonly used antiestrogen drug. It competes with estradiol for receptors and reduces the activity of cancer cells to inhibit their growth. Endocrine therapy is the first choice for postmenopausal patients with ER-positive breast cancer. Estrogen exerts a protective effect on cardiovascular function and can lower the blood pressure levels of female patients. Aromatase inhibitors represent an effective endocrine therapy for early and metastatic disease of hormone receptor-positive breast cancer, but by reducing the level of serum estrogen, they may also reduce the protective effect of estrogen on the cardiovascular system (34) (Figure 3). Therefore, when selecting endocrine therapy drugs for elderly patients, toxicity and side effects should be carefully evaluated as much as possible to avoid adverse effects on patients with accompanying diseases after comprehensive consideration of the patient's cardiovascular system, then a reasonable treatment plan should be developed.

\section{Targeted therapy-related cardiovascular events}

The main drugs used for breast cancer targeted drug therapy include the following categories: (I) anti-HER2 monoclonal antibodies, such as trastuzumab; (II) drugs targeting the P13K-ALK-mTOR pathway and other 
pathways, such as everolimus; (III) antiangiogenesis drugs; (IV) the CD4/6 inhibitor palbociclib; (V) polyadenosine diphosphate ribose polymerase inhibitor (PARPI); and (VI) drugs targeting the PD-1 pathway and immune surveillance mechanisms. The most common side effect of antiangiogenic drugs is hypertension.

\section{Antiangiogenic drugs}

The growth of tumors depends on an adequate blood supply. As solid tumors grow, the central core becomes hypoxic, which stimulates the growth and repair pathways of physiological tissues, including the release of angiogenic growth factors to allow the formation of new blood vessels. Vascular endothelial growth factor (VEGF) plays an important role in this process, and its inhibition has become the main therapeutic target in cancer treatment (35). Hypertension caused by antiangiogenesis targeted antitumor drugs is an important direction of tumor cardiology research (36). Antiangiogenic drugs mainly refer to VEGF signaling pathway inhibitors, including VEGF-A monoclonal antibodies, VEGF inhibitors (VEGF traps), VEGF receptor monoclonal antibodies, and small molecule tyrosine kinase inhibitors (TKIs). The incidence of hypertension caused by these drugs is $11-45 \%$, among which the incidence of severe grade 3-4 hypertension (CTCAE grade 3-4 for tumor adverse events, namely, systolic blood pressure $\geq 160$ and/or diastolic blood pressure $\geq 100 \mathrm{mmHg}$, or life-threatening, requiring emergency intervention) is $2-20 \%$. A study followed by Wildiers and others for 3 years showed that the most frequent grade $3-4$ adverse events were hypertension [in $6(15 \%)$ of 39 patients in the trastuzumab and pertuzumab group vs. $5(12 \%)$ of 41 in the trastuzumab and pertuzumab plus metronomic oral cyclophosphamide group] (37). A meta-analysis showed that TKIs increase the risk of serious CVD, particularly the risk of adverse events such as thrombosis and hypertension (38). VEGF inhibitors may increase the risk of heart failure, coronary heart disease, hypertension, and thromboembolic diseases through mechanisms such as endothelial injury, vasoconstriction and remodeling, inflammation, and platelet activation (39) (Figure 3). Endothelin-1 (ET-1), nitric oxide (NO), and VWF are all active substances that reflect the function of the vascular endothelium and have important roles as markers of the occurrence and development of CVD and primary hypertension. When hypertension occurs, the level of NO will decrease, and ET-1 and VWF levels will increase rapidly. NO is a key factor contributing to the vascular physiological changes of smooth muscle relaxation caused by eNOS. It plays an important role in angiogenesis. Normal endothelial cells constitutively express eNOS. When eNOS is inhibited under pathological conditions, the bioavailability of NO decreases (40).

\section{The mechanism of hypertension caused by TKIs}

TKIs are effective signal transduction inhibitors and have achieved significant progress in cancer treatment (41). By inhibiting vascular endothelial growth factor receptor (VEGFR)-mediated tumor blood vessel growth, VEGFRTKIs have become the main treatment for many solid malignant tumors $(42,43)$. However, the incidence of VEGFR-TKI-related cardiovascular toxicity is high and is not yet completely understood. VEGFR-TKIs can cause hypertension, left ventricular systolic dysfunction/ heart failure, and atherosclerotic thrombosis, as well as prolonged QT intervals and arrhythmia (44). Hypertension is a first-order effect of VEGFR-TKI treatment and the most common manifestation of cardiovascular toxicity. Almost every trial reported a treatment-related increase in blood pressure, with up to $80 \%$ of patients experiencing hypertension, or a recurrence or worsening of the previously controlled increase in blood pressure (45). VEGFR-TKIrelated hypertension may be severe and difficult to treat, but it is a dose-dependent and reversible process after stopping VEGFR-TKIs (46). However, the early identification of VEGFR-TKI-related hypertension and prompt initiation of treatment are still the main strategies.

The mechanism of hypertension during VEGFRTKI treatment is not completely understood. Decreased bioavailability of $\mathrm{NO}$ is a potentially important factor in VEGFR-TKI-related hypertension. VEGF stimulates the release of NO, and VEGF inhibition is related to a reduction in NO production. EGFR-TKI treatment is also related to an increase in the level of the strong vasoconstrictor ET-1. ET-1 receptor antagonists effectively reverse acute VEGFR-TKI-related hypertension (47). According to recent studies, oxidative stress is another mechanism by which VEGFR-TKIs induce vascular dysfunction in hypertension (48). In addition, studies have confirmed that the RhoA/ROCK pathway plays an important role in the onset of salt-sensitive hypertension and the occurrence of hypertension leading to myocardial hypertrophy. The vascular contraction effect of ET-1 is enhanced by the RhoA/ROCK pathway, and ET-1 activation participates in strengthening oxidative stress, promoting peroxide production, reducing endothelial NO production, and reducing vasodilation (49). 


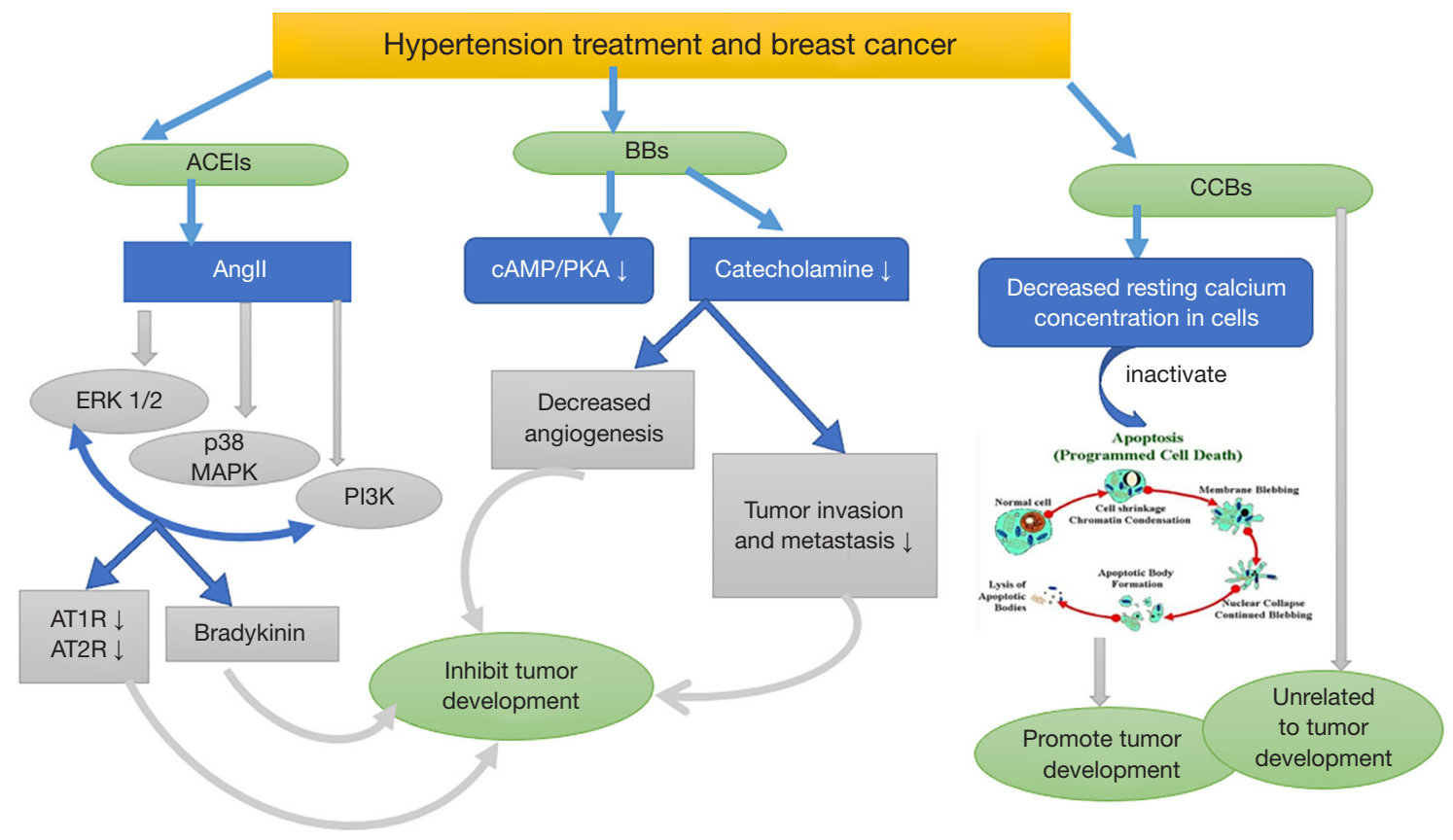

Figure 4 The relationship between antihypertensive drugs that including ACEIs, BBs, CCBs and breast cancer. ACEIs, angiotensinconverting enzyme inhibitors; BBs, $\beta$-blockers; CCBs, calcium channel blockers; cAMP, Cyclic Adenosine monophosphate; PKA, protein kinase A system; ERK1/2, extracellular regulated protein kinases 1/2; MAPK, mitogen-activated protein kinase; PI3K, phosphoinositide 3-kinase; AT1R, Angiotensin II Type 1 Receptor; AT1R, Angiotensin II Type 2 Receptor.

Therefore, the RhoA/ROCK pathway may be involved in the occurrence of hypertension caused by VEGF pathway inhibitors.

\section{The relationship between antihypertensive drugs and breast cancer}

Overall, the evidence indicates that the degree of blood pressure reduction is the main determinant of health benefits, and antihypertensive treatment has been shown to reduce the adverse effects of hypertension on CVD $(50,51)$. In view of the wide application of antihypertensive drugs, some studies have reported a correlation between hypertension, antihypertensive drugs, and breast cancer. However, the conclusions of these studies are not consistent. A correlation between antihypertensive drugs and breast cancer has been observed in premenopausal and postmenopausal women. A large number of studies have shown that a sharp decrease in estrogen levels plays an important role in the incidence of hypertension in postmenopausal women. With the support of the ESC Practice Guidelines Committee, the 2016 ESC position paper on cancer treatment and cardiovascular toxicity provided recommendations on the treatment of hypertension caused by tumor treatment, and angiotensinconverting enzyme inhibition was shown to improve the long-term prognosis of patients treated with angiotensinconverting enzyme inhibitors (ACEIs), angiotensin II receptor blockers (ARBs), and $\beta$-blockers (BBs) (36).

\section{ACEIs}

The renin-angiotensin system (RAS) is mainly involved in regulating arterial pressure $(52,53)$. Angiotensin II (AngII) is a bioactive peptide of the RAS, a major regulator of blood pressure and cardiovascular homeostasis, and a powerful mitogen. In addition to plasma AngII, the local RAS has been shown to function in different organs, leading to paracrine or autocrine functions of AngII. The roles of AngII in cell proliferation and migration and in some experimental models of angiogenesis suggest that this peptide may also be associated with cancer (54). AngII and advanced glycation end products (AGEs) activate ERK1/2, p38 MAPK, PI3K, and other signal transduction pathways by binding to their receptors (Figure 4). RAS includes angiotensin (AT) 2, angiotensin converting 
enzyme (ACE), AT [1-7], AT receptors 1 and 2 (AT1R and AT2R), and bradykinin and its receptors. High expression of AT1Rs and AT2Rs in tumor tissues promotes tumor progression (Figure 4). Both ARBs and ACEIs can reverse AT2-mediated angiogenesis and mitosis in tumor tissues. ACE2 expression reduces the production of VEGF and AT1R (1). AT1R signaling activates some tyrosine kinases, including epidermal growth factor receptor (EGFR), thereby stimulating the proto-cancer pathway (55). Related research shows that AT1R is overexpressed in breast hyperplasia and ductal carcinoma (intervertebral disc) and disappears in invasive breast cancer cells (56). AT2R is expressed at low levels in normal breast ducts, but its expression is increased in breast hyperplasia, intervertebral discs, and aggressive breast cancer (57). Other data indicate that the local RAS system produces AT2, which induces the epithelial-mesenchymal transition, tumor proliferation, and angiogenesis $(58,59)$. Therefore, the use of ACEIs in patients with breast cancer complicated by hypertension may reduce the recurrence rate of breast cancer and improve the long-term survival rate of patients with breast cancer.

\section{Calcium channel blockers (CCBs)}

CCBs are a group of antihypertensive drugs used to treat CVDs such as hypertension and angina pectoris. Although CCBs are widely used, people have long been concerned that CCBs may allow cancer cells to escape apoptosis by multiplication, leading to malignant growth $(60,61)$. In vitro and animal models show that CCBs reduce the resting calcium concentration in cells and inhibit the expression of apoptosis-related genes (62) (Figure 4). Although experimental evidence has shown that CCBs may lead to the growth of malignant tumors $(63,64)$, the study by Busby et al. of 23,669 patients with breast cancer did not find any association between drug use and breast cancer mortality. Therefore, to a certain extent, the increase in user deaths reported in previous studies may have been due to confusing instructions and other sources of bias, indicating that CCBs are safe prescriptions (65). However, the debate about the potential risks of CCBs has persisted for many years. A study of 79,4533 female patients aged 55 years and older in Taiwan conducted from 2001 to 2011 showed an increased risk of breast cancer among patients using all types of antihypertensive drugs, but patients with a cumulative use of more than 6 years did not present an increased risk. Even with dihydropyridine (DHP) and CCBs, no duration-response relationship has been found (66). Therefore, regarding its clinical significance, doctors should not sacrifice the long-term cardiovascular benefits of antihypertensive drugs simply because they are worried about the breast cancer risk that is still uncertain, and they should base their judgement on a consideration of multiple clinical factors.

\section{$B B s$}

Tumor proliferation is a complex multistep process, and BBs inhibit the proliferation of various breast cancer cell lines through the classical $\beta$-AR/CAMP/PKA pathway (Figure 4). Some epidemiological studies have shown that BBs prevent the progression of breast cancer. The mechanism may involve inhibiting the recruitment of macrophages and angiogenesis in the primary tumor, thereby affecting the progression of breast cancer $(67,68)$. Another study described an important role for the $\beta$-AR signaling pathway in the metastasis of breast cancer. Both stress and drug stimulation of $\beta$-AR cause tumors to metastasize up to 30 times (including lymph nodes and lungs). Selective BBs inhibit this prometastatic effect by $50 \%$ to $100 \%$. In addition, a meta-analysis showed that BBs may reduce the recurrence of breast cancer (8).

Stress associated with the neuroendocrine pathway may play a role in cancer progression (69). The stress hormone catecholamine is a potent norepinephrine receptor agonist and an inducer of cancer cell migration (70). Human breast cancer cells express $\beta$-adrenergic receptors (71). Meanwhile, catecholamine stimulation has been shown to promote angiogenesis, tumor invasion, and metastasis, and inhibits apoptosis in preclinical studies of various cancers, whereas BBs inhibit apoptosis of these cells, indicating that BBs inhibit norepinephrine-induced breast cancer metastasis $(72,73)$ (Figure 4). However, meta-analyses have shown that $\mathrm{BBs}$ are not beneficial in reducing the total number of deaths and cancer-specific deaths or breast cancer recurrence (74). Further research is needed to assess the effects of BBs on breast cancer and its subtypes.

A meta-analysis showed that BBs reduce the risk of breast cancer recurrence, and ACEIs and CCBs are not associated with an increased breast cancer risk (8). However, the current evidence reveals a complex relationship between antihypertensive drugs and cancer-related death. In particular, controversy exists regarding the risk of breast cancer in patients with long-term use of CCBs or ACEIs. A large 12-year clinical controlled trial showed that the use of 
CCBs by elderly patients with hypertension for 2-12 years was not associated with an increased risk of invasive breast cancer compared with patients who used CCBs for 1-2 years. Compared with patients taking ACEIs for 1-2 years, ACEIs exerted a protective effect on breast cancer in patients taking these drugs for 2-12 years. Specifically, the long-term use of CCBs did not increase the risk of breast cancer, and long-term use of ACEI treatment might have prevented breast cancer (75). Another report showed that CCBs inhibit the function of $T$ cells and macrophages (76). Moreover, the immunosuppressive effect of CCBs not only affects lymphocytes in the blood but also lymphocytes in the immune microenvironment (77). Therefore, the use of CCBs is not beneficial for patients with breast cancer complicated with hypertension, which contradicts the recommendation of the guidelines, and more clinical data are needed for further verification.

\section{Conclusions}

Hypertension is one of the most important adverse reactions in the treatment of breast cancer. It restricts its drug application and may affect the prognosis of patients. However, the pathogenesis of this type of hypertension is not the same as that of essential hypertension, and the specific mechanism is still unclear. No specific prevention and treatment drugs. Apatinib is a small molecule TKI of the VEGF pathway. Exploring the pathogenesis of apatinibinduced hypertension is essential for preventing related cardiovascular events and improving the prognosis of breast cancer patients. The endothelial function damage and oxidative stress mediated by the RhoA/ROCK pathway are involved in the pathogenesis of salt-sensitive hypertension, and the two may be involved in the same pathogenesis. Therefore, we speculate that the RhoA/ROCK pathway may be involved in the occurrence of hypertension caused by apatinib, and ROCK pathway inhibitors may have therapeutic effects on it.

A certain correlation has been identified between high blood pressure and cancers in specific tissues and specific pathological forms, of which breast cancer is more closely related to hypertension. Breast cancer and hypertension are causal factors for each other and form a vicious circle. Hypertension and cancer may share a common mechanism. The screening and risk assessment of breast cancer in patients with hypertension must be strengthened, and further studies of the common mechanisms of hypertension and cancer, such as inflammation, oxidative stress, ROS, and hormones, are needed. At the same time, severe hypertension caused by breast cancer treatment becomes an obstacle that prevents patients from continuing treatment. The biological mechanisms, molecular channels, and other factors associated with clinical treatment must be identified. Breast cancer cardiology is the interdisciplinary study of oncology and cardiology, and in-depth research in this field may result in long-term improvements in the survival and prognosis of patients with both clinical hypertension and breast cancer.

\section{Acknowledgments}

Funding: This study was supported by National Natural Science Foundation of China (NSFC-81960086, NSFC81670385 to Jing Yu).

\section{Footnote}

Reporting Checklist: The authors have completed the Narrative Review reporting checklist. Available at http:// dx.doi.org/10.21037/atm-21-2133

Conflicts of Interest: All authors have completed the ICMJE uniform disclosure form (available at http://dx.doi. org/10.21037/atm-21-2133). The authors have no conflicts of interest to declare.

Ethical Statement: The authors are accountable for all aspects of the work in ensuring that questions related to the accuracy or integrity of any part of the work are appropriately investigated and resolved.

Open Access Statement: This is an Open Access article distributed in accordance with the Creative Commons Attribution-NonCommercial-NoDerivs 4.0 International License (CC BY-NC-ND 4.0), which permits the noncommercial replication and distribution of the article with the strict proviso that no changes or edits are made and the original work is properly cited (including links to both the formal publication through the relevant DOI and the license). See: https://creativecommons.org/licenses/by-nc-nd/4.0/.

\section{References}

1. Aydiner A, Ciftci R, Sen F. Renin-Angiotensin system blockers may prolong survival of metastatic non-small cell lung cancer patients receiving erlotinib. Medicine 
2015;94:e887.

2. Weaver KE, Foraker RE, Alfano CM, et al. Cardiovascular risk factors among long-term survivors of breast, prostate, colorectal, and gynecologic cancers: a gap in survivorship care? J Cancer Surviv 2013;7:253-61.

3. Koene RJ, Prizment AE, Blaes A, et al. Shared Risk Factors in Cardiovascular Disease and Cancer. Circulation 2016;133:1104-14.

4. Pereira A, Garmendia ML, Alvarado ME, et al. Hypertension and the risk of breast cancer in Chilean women: a case-control study. Asian Pac J Cancer Prev 2012;13:5829-34.

5. Iacovelli R, Verri E, Cossu Rocca M, et al. The incidence and relative risk of cardiovascular toxicity in patients treated with new hormonal agents for castration-resistant prostate cancer. Eur J Cancer 2015;51:1970-7.

6. Ferlay J, Soerjomataram I, Dikshit R, et al. Cancer incidence and mortality worldwide: sources, methods and major patterns in GLOBOCAN 2012. Int J Cancer 2015;136:E359-86.

7. Radišauskas R, Kuzmickienè I, Milinavičienė E, et al. Hypertension, serum lipids and cancer risk: A review of epidemiological evidence. Medicina (Kaunas) 2016;52:89-98.

8. Podkowa N, Komasińska P, Roszak M, et al. Health behaviours among women diagnosed with breast tumours. Pol Merkur Lekarski 2014;37:153-8.

9. Han H, Guo W, Shi W, et al. Hypertension and breast cancer risk: a systematic review and meta-analysis. Sci Rep 2017;7:44877.

10. Esposito K, Chiodini P, Colao A, et al. Metabolic syndrome and risk of cancer: a systematic review and metaanalysis. Diabetes Care 2012;35:2402-11.

11. Feldman RD. Heart Disease in Women: Unappreciated Challenges, GPER as a New Target. Int J Mol Sci 2016;17:760.

12. Park NJ, Chang Y, Bender C, et al. Cardiovascular disease and mortality after breast cancer in postmenopausal women: Results from the Women's Health Initiative. PloS One 2017;12:e0184174.

13. Schneider AP, Zainer CM, Kubat CK, et al. The breast cancer epidemic: 10 facts. Linacre Q 2014;81:244-77.

14. Peeters PH, van Noord PA, Hoes AW, et al. Hypertension and breast cancer risk in a 19-year follow-up study (the DOM cohort). Diagnostic investigation into mammarian cancer. J Hypertens 2000;18:249-54.

15. Jarari N, Rao N, Peela JR, et al. A review on prescribing patterns of antihypertensive drugs. Clin Hypertens
2016;22:7.

16. Sieri S, Chiodini P, Agnoli C, et al. Dietary fat intake and development of specific breast cancer subtypes. J Natl Cancer Inst 2014;106:dju068.

17. Seretis A, Cividini S, Markozannes G, et al. Association between blood pressure and risk of cancer development: a systematic review and meta-analysis of observational studies. Sci Rep 2019;9:8565.

18. Zhao Y, Wang Q, Zhao X, et al. Effect of antihypertensive drugs on breast cancer risk in female hypertensive patients: Evidence from observational studies. Clin Exp Hypertens 2018;40:22-7.

19. McMaster WG, Kirabo A, Madhur MS, et al. Inflammation, immunity, and hypertensive end-organ damage. Circ Res 2015;116:1022-33.

20. Zahid H, Simpson ER, Brown KA. Inflammation, dysregulated metabolism and aromatase in obesity and breast cancer. Curr Opin Pharmacol 2016;31:90-6.

21. Nelson ER, Wardell SE, Jasper JS, et al. 27-Hydroxycholesterol links hypercholesterolemia and breast cancer pathophysiology. Science 2013;342:1094-8.

22. Thanan R, Oikawa S, Hiraku Y, et al. Oxidative stress and its significant roles in neurodegenerative diseases and cancer. Int J Mol Sci 2014;16:193-217.

23. Chen S, Parmigiani G. Meta-analysis of BRCA1 and BRCA2 penetrance. J Clin Oncol 2007;25:1329-33.

24. Antoniou A, Pharoah PDP, Narod S, et al. Average risks of breast and ovarian cancer associated with BRCA1 or BRCA2 mutations detected in case Series unselected for family history: a combined analysis of 22 studies. Am J Hum Genet 2003;72:1117-30.

25. Mai PL, Chatterjee N, Hartge P, et al. Potential excess mortality in BRCA1/2 mutation carriers beyond breast, ovarian, prostate, and pancreatic cancers, and melanoma. PloS One 2009; 4:e4812.

26. Arts-de Jong M, Maas AHEM, Massuger LF, et al. BRCA1/2 mutation carriers are potentially at higher cardiovascular risk. Crit Rev Oncol Hematol 2014;91:159-71.

27. van Westerop LLM, Arts-de Jong M, Hoogerbrugge N, et al. Cardiovascular risk of BRCA1/2 mutation carriers: A review. Maturitas 2016;91:135-9.

28. Matsubayashi J, Takanashi M, Oikawa K, et al. Expression of $\mathrm{G}$ protein-coupled receptor kinase 4 is associated with breast cancer tumourigenesis. J Pathol 2008;216:317-27.

29. Rayner B, Ramesar R. The importance of $G$ proteincoupled receptor kinase 4 (GRK4) in pathogenesis of salt sensitivity, salt sensitive hypertension and response to 
antihypertensive treatment. Int J Mol Sci 2015;16:5741-9.

30. Liu C, Xi B. Pooled analyses of the associations of polymorphisms in the GRK4 and EMILIN1 genes with hypertension risk. Int J Med Sci 2012;9:274-9.

31. Amir E, Seruga B, Niraula S, et al. Toxicity of adjuvant endocrine therapy in postmenopausal breast cancer patients: a systematic review and meta-analysis. J Natl Cancer Inst 2011;103:1299-309.

32. Hozumi Y, Suemasu K, Takei H, et al. The effect of exemestane, anastrozole, and tamoxifen on lipid profiles in Japanese postmenopausal early breast cancer patients: final results of National Surgical Adjuvant Study BC 04, the TEAM Japan sub-study. Ann Oncol 2011;22:1777-82.

33. Ewer MS, Glück S. A woman's heart: the impact of adjuvant endocrine therapy on cardiovascular health. Cancer 2009;115:1813-26.

34. Foglietta J, Inno A, de Iuliis F, et al. Cardiotoxicity of Aromatase Inhibitors in Breast Cancer Patients. Clin Breast Cancer 2017;17:11-7.

35. Simons M, Gordon E, Claesson-Welsh L. Mechanisms and regulation of endothelial VEGF receptor signalling. Nat Rev Mol Cell Biol 2016;17:611-25.

36. Zamorano JL, Lancellotti P, Rodriguez Muñoz D, et al. 2016 ESC Position Paper on cancer treatments and cardiovascular toxicity developed under the auspices of the ESC Committee for Practice Guidelines: The Task Force for cancer treatments and cardiovascular toxicity of the European Society of Cardiology (ESC). Eur Heart J 2016;37:2768-801.

37. Wildiers H, Tryfonidis K, Dal Lago L, et al. Pertuzumab and trastuzumab with or without metronomic chemotherapy for older patients with HER2-positive metastatic breast cancer (EORTC 75111-10114): an open-label, randomised, phase 2 trial from the Elderly Task Force/Breast Cancer Group. Lancet Oncol 2018;19:323-36.

38. Totzeck M, Mincu R-I, Mrotzek S, et al. Cardiovascular diseases in patients receiving small molecules with antivascular endothelial growth factor activity: A meta-analysis of approximately 29,000 cancer patients. Eur J Prev Cardiol 2018;25:482-94.

39. Chen XL, Lei YH, Liu CF, et al. Angiogenesis inhibitor bevacizumab increases the risk of ischemic heart disease associated with chemotherapy: a meta-analysis. PloS One 2013;8:e66721.

40. Kubota N, Miyauchi K, Kasai T, et al. Synergistic effects of calcium-channel and angiotensin-receptor blockers on endothelial function and inflammatory responses in a porcine drug-eluting stent model. Circ J 2010;74:1704-10.

41. Liu K, Ren T, Huang Y, et al. Apatinib promotes autophagy and apoptosis through VEGFR2/STAT3/BCL2 signaling in osteosarcoma. Cell Death Dis 2017;8:e3015.

42. Dobbin SJH, Cameron AC, Petrie MC, et al. Toxicity of cancer therapy: what the cardiologist needs to know about angiogenesis inhibitors. Heart 2018;104:1995-2002.

43. Li F, Zhu T, Cao B, et al. Apatinib enhances antitumour activity of EGFR-TKIs in non-small cell lung cancer with EGFR-TKI resistance. Eur J Cancer 2017;84:184-92.

44. Ghatalia P, Je Y, Kaymakcalan MD, et al. QTc interval prolongation with vascular endothelial growth factor receptor tyrosine kinase inhibitors. Br J Cancer 2015;112:296-305.

45. Small HY, Montezano AC, Rios FJ, et al. Hypertension due to antiangiogenic cancer therapy with vascular endothelial growth factor inhibitors: understanding and managing a new syndrome. Can J Cardiol 2014;30:534-43.

46. Robinson ES, Matulonis UA, Ivy P, et al. Rapid development of hypertension and proteinuria with cediranib, an oral vascular endothelial growth factor receptor inhibitor. Clin J Am Soc Nephrol 2010;5:477-83.

47. Kappers MH, de Beer VJ, Zhou Z, et al. Sunitinib-induced systemic vasoconstriction in swine is endothelin mediated and does not involve nitric oxide or oxidative stress. Hypertension 2012;59:151-7.

48. Neves KB, Rios FJ, van der Mey L, et al. VEGFR (Vascular Endothelial Growth Factor Receptor) Inhibition Induces Cardiovascular Damage via Redox-Sensitive Processes. Hypertension 2018;71:638-47.

49. Tsai SH, Lu G, Xu X, et al. Enhanced endothelin-1/Rhokinase signalling and coronary microvascular dysfunction in hypertensive myocardial hypertrophy. Cardiovasc Res 2017;113:1329-37.

50. Zanchetti A, Thomopoulos C, Parati G. Randomized controlled trials of blood pressure lowering in hypertension: a critical reappraisal. Circ Res 2015;116:1058-73.

51. Brook RD, Kaciroti N, Bakris G, et al. Prior Medications and the Cardiovascular Benefits From Combination Angiotensin-Converting Enzyme Inhibition Plus Calcium Channel Blockade Among High-Risk Hypertensive Patients. J Am Heart Assoc 2018;7:e006940.

52. Li P, Kondo T, Numaguchi Y, et al. Role of bradykinin, nitric oxide, and angiotensin II type 2 receptor in imidapril-induced angiogenesis. Hypertension 2008;51:252-8.

53. Tom B, Dendorfer A, Danser AHJ. Bradykinin, 
angiotensin-(1-7), and ACE inhibitors: how do they interact? Int J Biochem Cell Biol 2003;35:792-801.

54. Deshayes F, Nahmias C. Angiotensin receptors: a new role in cancer? Trends Endocrinol Metab 2005;16:293-9.

55. Eguchi S, Inagami T. Signal transduction of angiotensin II type 1 receptor through receptor tyrosine kinase. Regul Pept 2000;91:13-20.

56. De Paepe B, Verstraeten VL, De Potter CR, et al. Growth stimulatory angiotensin II type-1 receptor is upregulated in breast hyperplasia and in situ carcinoma but not in invasive carcinoma. Histochem Cell Biol 2001;116:247-54.

57. De Paepe B, Verstraeten VM, De Potter CR, et al. Increased angiotensin II type-2 receptor density in hyperplasia, DCIS and invasive carcinoma of the breast is paralleled with increased iNOS expression. Histochemistry and cell biology 2002;117:13-9.

58. Feng Y, Wan H, Liu J, et al. The angiotensin-converting enzyme 2 in tumor growth and tumor-associated angiogenesis in non-small cell lung cancer. Oncol Rep 2010;23:941-8.

59. Qian YR, Guo Y, Wan HY, et al. Angiotensin-converting enzyme 2 attenuates the metastasis of non-small cell lung cancer through inhibition of epithelial-mesenchymal transition. Oncol Rep 2013;29:2408-14.

60. Mason RP. Calcium channel blockers, apoptosis and cancer: is there a biologic relationship? J Am Coll Cardiol 1999;34:1857-66.

61. Hanahan D, Weinberg RA. The hallmarks of cancer. Cell 2000;100:57-70.

62. Jang SJ, Choi HW, Choi DL, et al. In vitro cytotoxicity on human ovarian cancer cells by T-type calcium channel blockers. Bioorg Med Chem Lett 2013;23:6656-62.

63. Zhao T, Guo D, Gu Y, et al. Nifedipine stimulates proliferation and migration of different breast cancer cells by distinct pathways. Mol Med Rep 2017;16:2259-63.

64. Guo DQ, Zhang H, Tan SJ, et al. Nifedipine promotes the proliferation and migration of breast cancer cells. PloS One 2014;9:e113649.

65. Busby J, Mills K, Zhang SD, et al. Postdiagnostic Calcium Channel Blocker Use and Breast Cancer Mortality: A Population-based Cohort Study. Epidemiology 2018;29:407-13.

66. Chang CH, Chiang CH, Yen CJ, et al. Antihypertensive agents and the risk of breast cancer in women aged 55 years and older: a nested case-control study. J Hypertens 2016;34:558-66.
67. Melhem-Bertrandt A, Chavez-Macgregor M, Lei X, et al. Beta-blocker use is associated with improved relapse-free survival in patients with triple-negative breast cancer. J Clin Oncol 2011;29:2645-52.

68. Powe DG, Voss MJ, Zänker KS, et al. Beta-blocker drug therapy reduces secondary cancer formation in breast cancer and improves cancer specific survival. Oncotarget 2010;1:628-38.

69. Antoni MH, Lutgendorf SK, Cole SW, et al. The influence of bio-behavioural factors on tumour biology: pathways and mechanisms. Nat Rev Cancer 2006;6:240-8.

70. Masur K, Niggemann B, Zanker KS, et al. Norepinephrine-induced migration of SW 480 colon carcinoma cells is inhibited by beta-blockers. Cancer research 2001;61:2866-9.

71. Vandewalle B, Revillion F, Lefebvre J. Functional betaadrenergic receptors in breast cancer cells. J Cancer Res Clin Oncol 1990;116:303-6.

72. Sloan EK, Priceman SJ, Cox BF, et al. The sympathetic nervous system induces a metastatic switch in primary breast cancer. Cancer Res 2010;70:7042-52.

73. Vaklavas C, Chatzizisis YS, Tsimberidou AM. Common cardiovascular medications in cancer therapeutics. Pharmacol Ther 2011;130:177-90.

74. Kim HY, Jung YJ, Lee SH, et al. Is Beta-Blocker Use Beneficial in Breast Cancer? A Meta-Analysis. Oncology 2017;92:264-8.

75. Raebel MA, Zeng C, Cheetham TC, et al. Risk of Breast Cancer With Long-Term Use of Calcium Channel Blockers or Angiotensin-Converting Enzyme Inhibitors Among Older Women. Am J Epidemiol 2017;185:264-73.

76. Matsumori A, Nishio R, Nose Y. Calcium channel blockers differentially modulate cytokine production by peripheral blood mononuclear cells. Circ J 2010;74:567-71.

77. Takada K, Kashiwagi S, Asano Y, et al. Verification of the effects of calcium channel blockers on the immune microenvironment of breast cancer. BMC Cancer 2019;19:615.

Cite this article as: Wang $\mathrm{W}$, He Q, Zhang H, Zhuang C, Wang Q, Li C, Sun R, Fan X, Yu J. A narrative review on the interaction between genes and the treatment of hypertension and breast cancer. Ann Transl Med 2021;9(10):894. doi: 10.21037/ atm-21-2133 